\title{
Differential Gain Comparison of Optical Planar Amplifier on Silica Glasses Doped with Bi-Ge and Er, Yb lons
}

\author{
Jiř́ Šmejcký゙ and Vítězslav Jeřábek
}

\section{Faculty of Electrical Engineering, Technical University in Prague}

\begin{abstract}
The paper presents the measurement and calculation of the optical amplifier gain and the optimal length of the active optical amplifier waveguide doped with $\mathrm{Bi}-\mathrm{Ge}$ radiation complexes compared to an optical amplifier with active doping with ions $\mathrm{Er}^{3+}, \mathrm{Yb}^{3+}$. At present are using optical amplifiers for the high-capacity optical communication systems in the narrow spectral region of $1530-1560 \mathrm{~nm}$, determined by the gain bandwidth of erbium-doped fiber amplifiers (EDFA) or erbium-doped optical planar amplifiers (EDPA) realized as active planar waveguides in the optical integrated circuits technique. However, it is possible increase wavelength region up to $1610 \mathrm{~nm}$, where optical losses of telecommunication fibers are less than $0.3 \mathrm{~dB}$ per $\mathrm{km}$, if appropriate amplifiers are available. In this regard, the development of novel optical amplifiers operating in this spectral region have of great importance. The paper summarizes the results measurement of the attenuation and emission spectra for net gain spectra calculation of the new ion exchange $\mathrm{Ag}^{+}-\mathrm{Na}^{+} \mathrm{optical} \mathrm{Er}^{3+}$ and $\mathrm{Yb}^{3+}$ doped active planar waveguides realized on silica glass substrates and parameters of novel optical amplifiers for extension of the bandwidth from 1530 to $1610 \mathrm{~nm}$ doped by bismuth - erbium ions.
\end{abstract}

Keywords: Optical waveguides, EDFA, EDPA, BEDF, BAC-Ge, luminescence spectrum.

\section{INTRODUCTION}

In optical telecommunication systems, optical amplifiers are one of the essential parts of the optical transmission tracts containing silicon fiber waveguides. The main part of optical amplifiers is an optically active fiber waveguide. The information capacity of an optical fiber transmission path is determined by the width of a spectrum of optical amplifiers that use the quantum principle of stimulated radiation and are extensively wave selective. Their selectivity depends on the type of activator that is used to amplify the passing radiation. In order to expand the transmission capacity of the connection, it is necessary to extend the bandwidth of the optical amplifiers. This is possible by using two or more activators in the optical fiber optic amplifier waveguide. In the last few years, several works have been published on broadband fiber active waveguides for optical amplifiers in telecommunication bands. These waveguides contain with erbium and bismuth ions [1], [2] for medium wavelength telecommunication bands at 1340, 1430 and $1700 \mathrm{~nm}$. Broadband optical transmission systems are currently being implemented with parallel optic amplifiers. However, the use of several separate optical amplifiers for broadband telecommunication systems is not optimal, both economically and technically. A more preferable solution would be to use a fiber or planar amplifier with a high bandwidth amplifier, for example for $\mathrm{S}+\mathrm{C}+\mathrm{L}$ $(1460-1615 \mathrm{~nm})$ or $\mathrm{C}+\mathrm{L}+\mathrm{U}(1530-1675 \mathrm{~nm})$ bands. Many attempts have been made to achieve optical amplification using erbium and bismuth codoped optical waveguides for broadband near-IR (NIR) band systems. Unfortunately, until now, the continuous

Address correspondence to this article at the Faculty of Electrical Engineering, Technical University in Prague; E-mail: J.Smejcky@seznam.cz spectral range of these materials has not been demonstrated in the desired wavelength range.

The problem is that the typical luminescence spectrum of bismuth and erbium-doped active waveguides has a slight overlap, especially for shorter wavelengths. For this reason, it is not possible to implement a broadband amplifier with a balanced amplification in the band $\mathrm{S}+\mathrm{C}+\mathrm{L}(1460-1615 \mathrm{~nm})$, where the overlap of the luminescence spectrum of erbium and bismuth is stronger, see Figure 1.

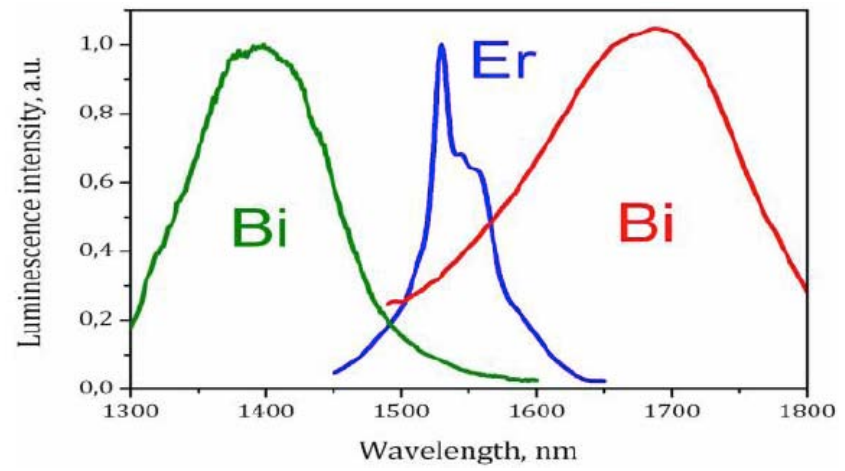

Figure 1: Typical luminescence spectrum of fiber doped with $\mathrm{Bi}$ and $\mathrm{Er}[3]$.

\section{INTRODUCTION TO BROADBAND OPTICAL WAVEGUIDE ISSUES}

At present, optical amplifiers for high-capacity optical communication systems in the narrow spectral range of 1530-1560 $\mathrm{nm}$ are used, which are determined by the erbium-doped fiber amplification bandwidth (EDFA) or erbium-doped optical planar amplifiers (EDPA) as active planar waveguides in the optical integrated circuits. However, when suitable amplifiers are available, it is possible to increase the range of wavelengths up to $1610 \mathrm{~nm}$, where the optical loss of telecommunication fibers is less than $0.3 \mathrm{~dB} /$ $\mathrm{km}$. In this respect, the development of new optical 


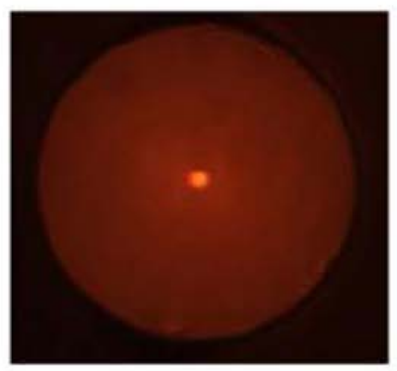

a

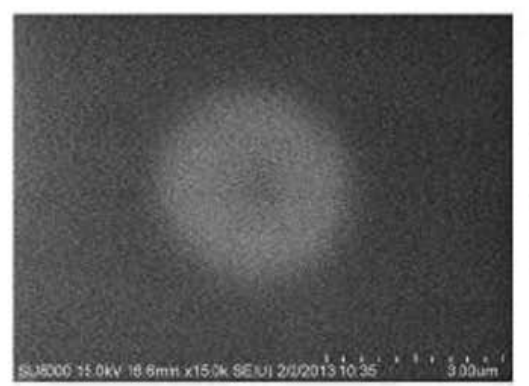

b

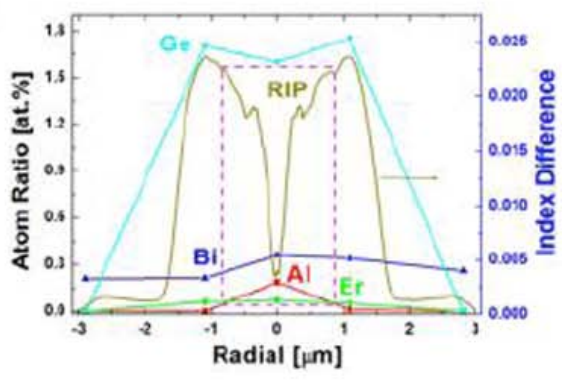

C

Figure 2: (a) Cross-sectional image of BEDF; b) image of the BEDF core region SEM; c) BEDF composite radial element and refractive index (RIP) profile (the dashed-line rectangle is the area of impact) [1].

amplifiers operating in this spectral range is of great importance.

In the study of [2], the output optical power of bismuth / erbium doped optical fiber (BEDF) was studied in double pumping at $830 \mathrm{~nm}$ and $980 \mathrm{~nm}$. Based on these experiments, the possible energy transitions of the BEDF were proposed. The measurement results demonstrate the benefits of pumping BEDF at $830 \mathrm{~nm}$ versus $980 \mathrm{~nm}$ in terms of absorption, pumping efficiency, excited state absorption and amplification. Since the first demonstration of broadband emission in glass doped with bismuth [4], attention has increasingly been paid to active Bismuth-doped waveguides for their potential use as active media for broadband amplifiers and super-luminescent sources in the spectral range of 1150-1800 nm. Bismuth-doped waveguides have a wavelength range of $1100-1600 \mathrm{~nm}$. Their fluorescence strongly depends on the wavelength of the pumping radiation because they contain several active centers (bismuth active centers, BACs) with different excitation and emission spectra. This makes it possible to adjust the excitation and emission wavelengths by a type of modifier such as $\mathrm{Al}, \mathrm{Si}, \mathrm{Ge}$ or $\mathrm{P}$. This feature allows you to fine-tune the power of the active bismuth-doped fibres (BDF) by selecting the wavelength of the pumping source. It also shows that BACs are very complicated in nature. It was also shown in [2] that the profile and bandwidth can also be selected and set by the appropriate choice of the source(s) wavelength. Due to the existence of more active bands in the BEDF waveguide, it is advantageous to carry out simultaneous pumping at multiple wavelengths. A comparison of the BEDF power output at the pumping wavelength of $830 \mathrm{~nm}$ and $980 \mathrm{~nm}$ was made. At that, waveguide attenuation, luminescence properties and on-off gain were measured. By analyzing absorption, amplification, and ESA at $830 \mathrm{~nm}$ and $980 \mathrm{~nm}$, two different energy conversion processes were designed and an attempt was made to explain the broadband emission in the BEDF.

\subsection{Measurement of the Fiber Waveguide}

The research study [1] describes the production of a BEDF waveguide on which the measurement was done, Figure 2.

In Figure $\mathbf{2} \mathbf{a}, \mathbf{b}$ is a cross section of the waveguide obtained with a scanning electron microscope. It can be observed that the waveguide can be considered rotationally symmetrical. Figure $\mathbf{2 c}$ is a profile of composite radial element with a plurality of additional dopants (germanium, phosphorus, aluminum, etc.) and wavelength core refractive index waveform (RIP).

\subsection{Absorption Spectral Characteristics}

On this active optical waveguide, the attenuation was first measured (Figure 3).

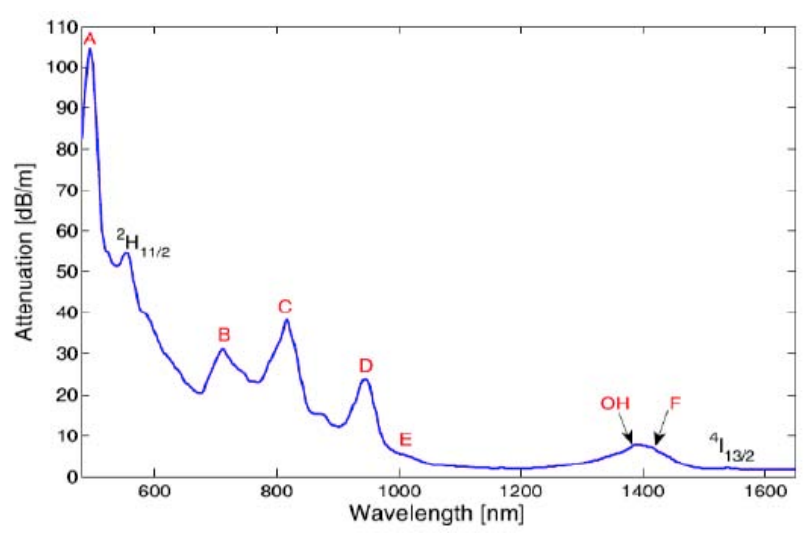

Figure 3: BEDF Attenuation Spectrum [1].

The graph of the spectral damping characteristics shows the spikes at points around 500 (A), 710 (B), $820(C), 950(D), 1000(E)$ and $1400 \mathrm{~nm}(\mathrm{~F})$, with peaks of 550 and $1530 \mathrm{~nm}$ being attributed to $\mathrm{Er}^{3+}$. Together $\mathrm{BAC}-\mathrm{Ge}, \mathrm{Bi}^{2+}$ and $\mathrm{BAC}-\mathrm{Si}$ are responsible for the attenuation bands around points $A, B, C$. Gaussian selection (filtering) distinguished three attenuation zones in the range of 920 to $1100 \mathrm{~nm}$. These are attenuation peaks around wavelengths $\sim 950, \sim 980$ and $\sim 1020 \mathrm{~nm}$ which are attributed to the absorption of $\mathrm{BAC}-\mathrm{Ge}, \mathrm{Er}^{3+}$ and BAC-AI. In addition, there are also 
three attenuation bands between 1100 and $1480 \mathrm{~nm}$, which are attenuations at $\sim 1350, \sim 1380$ and $\sim 1410$ $\mathrm{nm}$ for which BAC-P, OH and BAC-Si are responsible. Spectral Attenuation Characteristics shows the possibility of pumping BEDF at $830 \mathrm{~nm}$ (BAC-Si) and $980 \mathrm{~nm}$ (BAC-Al and $\mathrm{Er}^{3+}$ ). Additionally, the attenuation coefficient at $830 \mathrm{~nm}(30.9 \mathrm{~dB} / \mathrm{m})$ is approximately four times larger than at $980 \mathrm{~nm}(7.7 \mathrm{~dB} / \mathrm{m})$. It follows that the effective cross section of the absorption at $830 \mathrm{~nm}$ is larger than at $980 \mathrm{~nm}$.

\subsection{Emission Spectral Characteristics BEDF}

The emission spectrum of BEDF excited at $830 \mathrm{~nm}$ and $405 \mathrm{~nm}$ was measured. The normalized emission spectrum is shown in Figure 4.

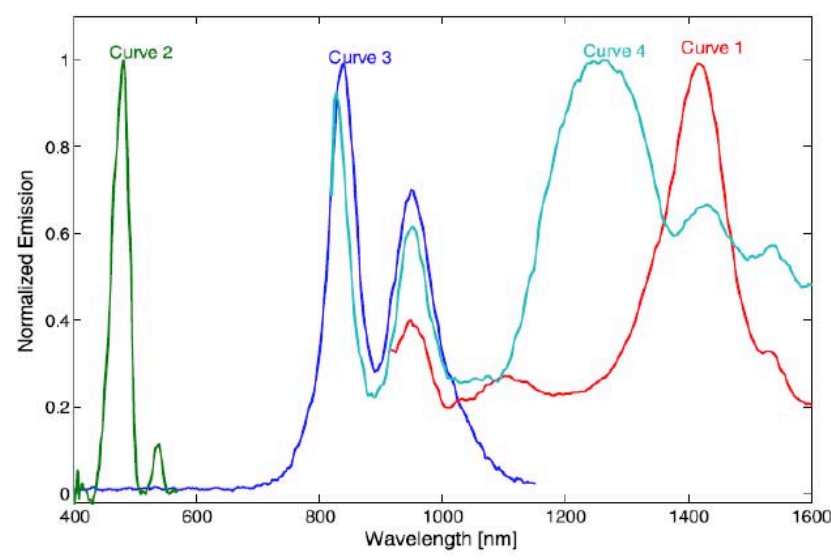

Figure 4: Normalized emission spectra BEDF excited at 830 nm (curve 1 and curve 2) and $405 \mathrm{~nm}$ (curve 3 and curve 4) [1].

From the normalized emission characteristics, the existence of the six NIR emission bands (curves 1,3 and 4) occurring around $\sim 830,950,1100,1260,1410$ and $1530 \mathrm{~nm}$ where the first five bands belong to the BAC emission, $1530 \mathrm{~nm}$ corresponds to the electron transitions of the erbium $\left({ }^{4} I_{13 / 2}=>{ }^{4} I_{15 / 2}\right)$. The emission bands at 830, 1410, 950, 1110 and $1260 \mathrm{~nm}$ correspond to BAC-Si, BAC-Ge, BAC-Al and BAC-P. In addition to NIR emissions, two UP-conversions [7] in the bands 480 and $540 \mathrm{~nm}$ (curve 2) are identifiable. In addition, the NIR lifetime that is measured at wavelengths $950,1110,1260$ and $1410 \mathrm{~nm}$ is approximately $110,150,450$ and $590 \mu \mathrm{s}$.

Measuring the life of metastable states corresponds well with the emission spectrum analysis and confirmed the existence of four BACs: BAC-Ge, BAC-AI, BAC-P and BAC-Si. The results of the measurements of the metastasis statuses indicate that the most stable is the metastable state of BAC-Si (590 $\mu s)$.

Comparison of emission spectral characteristics for single or double pumping. The measurements were made in the arrangement shown in Figure 5. The measured characteristics are shown in Figure 6.

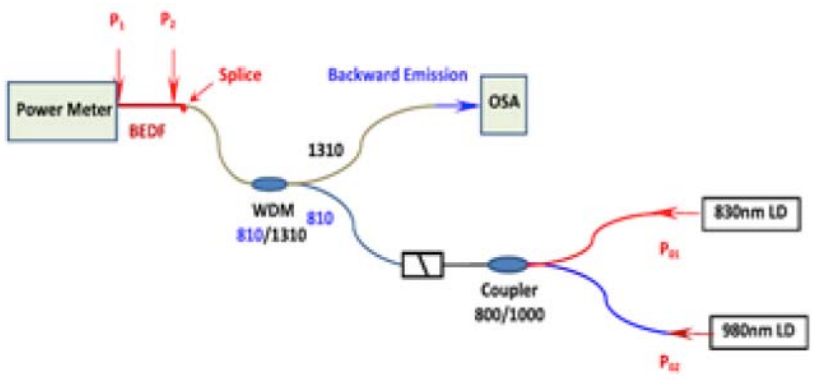

Figure 5: Experimental setup for the back ward emission measurement with $830 \mathrm{~nm}$ or $980 \mathrm{~nm}$ pumps.

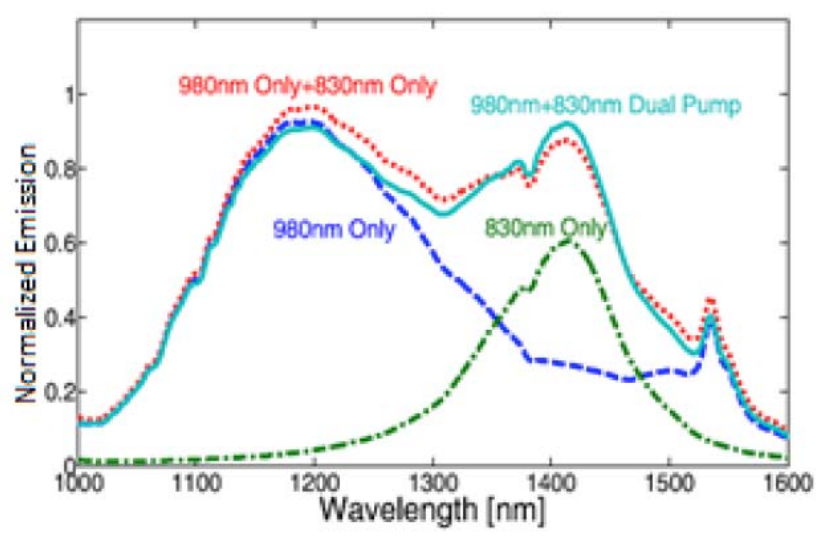

Figure 6: Experimental measurements of emissions while using $830 \mathrm{~nm}$ or $980 \mathrm{~nm}$ pumps, alternatively and concurrently [1].

In the experimental system, the Optical Spectrum Analyzer (OSA) was used to record backward emission spectra of a $50 \mathrm{~cm}$ long BEDF. Laser diodes $830 \mathrm{~nm}$ and $980 \mathrm{~nm}$ were connected via WDM 800/1000 nm multiplexer to the measured waveguide. The power meter was connected to the output of the waveguide to monitor the residual pumping power for luminescence analysis. From the graph in Figure 6 we can clearly see one emission band at $1410 \mathrm{~nm}$ (BAC-Si) when pumping only at $830 \mathrm{~nm}$. When pumping only at 980 $\mathrm{nm}$, there are two emission zones, namely 1190 for BAC-Al and BAC- $P$ and $1536 \mathrm{~nm}$ for $\mathrm{Er}^{3+}$. By way of comparison, Figure 6 shows the dependence of the emission on pumping power for pumping wavelengths of $830 \mathrm{~nm}$ and $980 \mathrm{~nm}$. It is clear that the emission at $830 \mathrm{~nm}$ pumping is growing much faster than when pumping at $980 \mathrm{~nm}$.

More detailed information and design of BEDF energy transfer diagram at pumping $980 \mathrm{~nm}$ and 830 $\mathrm{nm}$ is given in the work [1].

\subsection{Optical Amplifier for $C+L+U$ Telecommunication Bands}

The results of the experimental measurement by bismuth are remarkable. However, for the third optical 
window of quartz waveguides NIR, the band between $1500 \mathrm{~nm}$ and $1700 \mathrm{~nm}$ is needed. A fiber amplifier for $\mathrm{C}+\mathrm{L}+\mathrm{U}$ telecommunication area with continuous amplification in the band $1530-1675 \mathrm{~nm}$ is described In the study of [3]. In this case, germanosilicate fibers are co-doped with various contents of bismuth and erbium that have been produced by the modified chemical vapor deposition method (MCVD). It should be noted that the BAC emission parameters are strongly dependent on the manufacturing parameters. Therefore, the production of an active optical waveguide with a corresponding $\mathrm{Bi} / \mathrm{Er}$ ratio is one of the key issues. It is also known that the optical properties of the activated bismuth doped waveguide depend on the chemical composition of the glass from which they are made. An absorbtion spectrum of fibers with different $\mathrm{Bi} / \mathrm{Er}$ ratios is shown in the picture Figure 7.

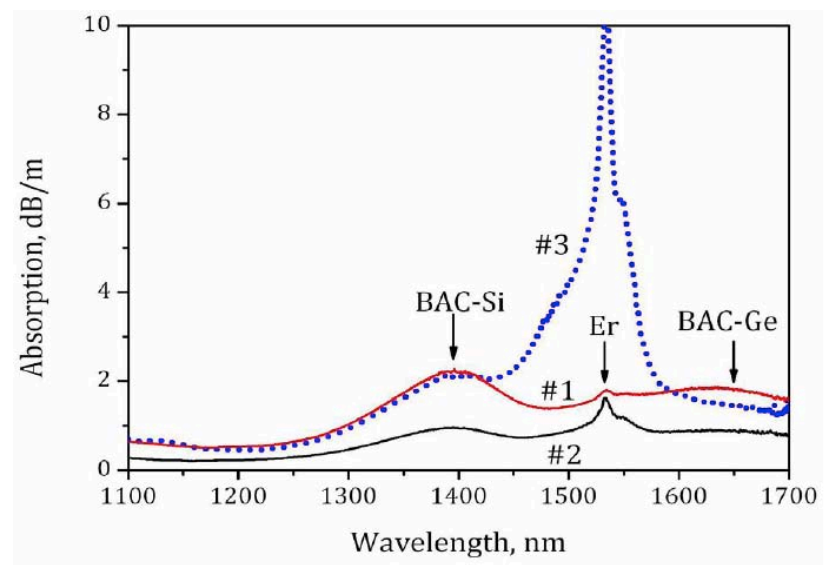

Figure 7: Absorption spectra of the various Bi/Er-codoped germanosilicate fibers [3].

The graphic waveforms exhibit two different absorption peaks at 1650 and $1400 \mathrm{~nm}$, which belong to BAC-Ge and BAC-Si. The absorption band with a peak at $1535 \mathrm{~nm}$ is assigned to $\mathrm{Er}^{3+}$ ions. It can be seen that the BAC-Ge and $\mathrm{Er}$ bands overlap significantly. This fact is also schematically shown in Figure 11, where the energy levels of $\mathrm{Er}^{3+}$ ion and BAC-Ge overlap. Therefore, for the excitation of both active centers, it is possible to use only one source of pumping radiation, in this case at a wavelength of 1460 $\mathrm{nm}$. This is an indisputable advantage over the use of two pumping sources. In this case, when using only one source of pumping radiation at $1460 \mathrm{~nm}$, a continuous gain in the range $1530-1675 \mathrm{~nm}$ was achieved, Figure 8. The technological parameters of the investigated fibers are listed in Table 1.

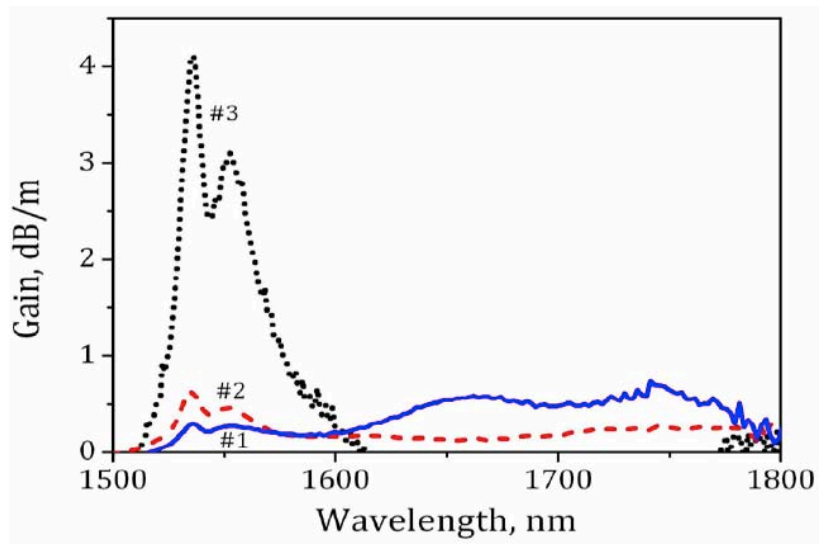

Figure 8: Net gain spectra of the investigated fibers at pumping1460 $\mathrm{nm}$ [3].

\section{THEORETICAL ANALYSIS OF THE OPTICAL ACTIVE WAVEGUIDE}

The theoretical analysis is based on a mathematical model by rate equations, that describes approximately the behavior of the optical active structures (fiber or planar waveguides) and allows to predict theoretically the quantum behavior of the structures and perform some optimizations on technological level. The behavior of the optical active structures is influenced by a number of factors, both the actual composition of the active material and its geometric configuration, the choice of pumping power, the active length of the waveguide. Finding optimal concentrations of activators and precursors is one of the pivotal challenges in the development of optically active structures. At higher concentrations of activators, luminescence is extinguished by clustering of the activator atoms, energy migration or cross-relaxation. The Judd-Ofelt theory is used to assess the influence of matrix atoms on the active ions (activators). However, this optimization is not the subject of this work.

In this work, a mathematical model will be described which allows for the optimization of the length of the active structure and the choice of the pumping power on the basis of solutions of rate equations and equation describing the propagation of the optical signal in the active optical waveguide.

Table 1: The Technological Characteristics of the Fibers [3]

\begin{tabular}{|c|c|c|c|c|}
\hline Fiber & Core glass, mol.\% & Bi, ppm & Active absorption at $\mathbf{1 6 5 0 ~} \mathbf{n m}, \mathbf{d B} \mathbf{m}^{-1}$ & $\mathbf{E r , p p m}$ \\
\hline \hline$\# 1$ & $\sim 50 \mathrm{GeO}_{2}-50 \mathrm{SiO}_{2}$ & 140 & 1.6 & 15 \\
\hline$\# 2$ & $\sim 50 \mathrm{GeO}_{2}-50 \mathrm{SiO}_{2}$ & 150 & 1.4 & 100 \\
\hline$\# 3$ & $\sim 50 \mathrm{GeO}_{2}-50 \mathrm{SiO}_{2}$ & 150 & 0,85 & 1500 \\
\hline
\end{tabular}


The calculation of the amplification of the optical active waveguide essentially resides in the solution of the system of differential equations describing the occupation of atomic levels (the so-called rate equations) and in the solution of propagation of light by the propagation equation. Differential rate equations are derived from three basic phenomena describing the probability of the transition of the quantum system: spontaneous emission, stimulated emission and absorption. The energy gradients in the erbium-ytterbium complex are described by absorption and emission effective cross-sections. For insulated non-degraded atoms, the following applies: $\sigma_{e}(v)=$ $\sigma_{a}(v)=\sigma(v)$. In the real system, however, the energy levels are always degenerated and therefore the absorption and emission spectral waveforms differ: $\left(\sigma_{e}(v) \neq \sigma_{a}(v)\right)$.

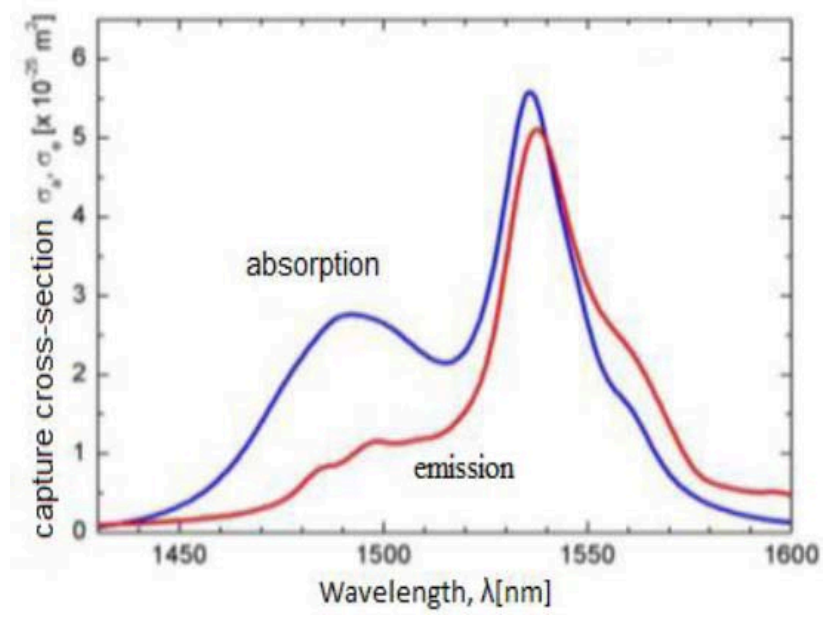

Figure 9: Absorption and emission cross-section spectrum of the silica waveguide $\mathrm{Er}^{3+}[5,7]$.

\subsection{Rate Equations for Three-Level System for Er and $\mathrm{Yb}$}

The Erbium-Ytterbium complex can roughly be described as a three-level quantum system (1) - (3) using rate equations in the form:

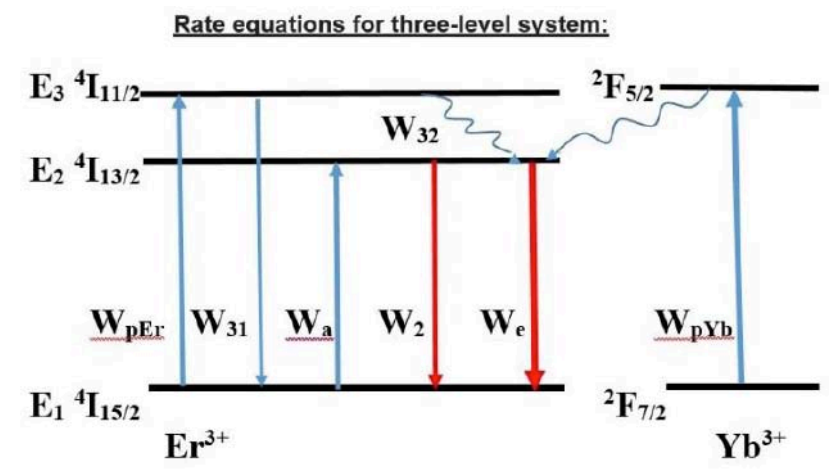

Figure 10: Erbium-Ytterbium complex can roughly be described as a three-level quantum system.

$\frac{d N_{3}}{d t}=-W_{32} N_{3}-W_{31} N_{3}+W_{p E r} N_{1}$

$$
\begin{aligned}
& \frac{d N_{2}}{d t}=W_{32} N_{3}-W_{2} N_{2}-W_{e} N_{2}+W_{a} N_{1}+W_{p} N_{1} \\
& N_{\text {tot }}^{E r+Y b}=N_{1}+N_{2}+N_{3}=N_{t o t}
\end{aligned}
$$

Where:

$N_{1}, N_{2}, N_{3}$ are the numbers of excited ions in a unit volume (ion concentration) at 1,2,3 levels $N_{\text {tot }}$ is the total number of excited ions in a unit volume

$W_{32}$ is the rate coefficient of non-radiation transition from $E_{3}$ to $E_{2}$

$W_{31}$ is the rate coefficient of non-radiation transition from $E_{3}$ to $E_{1}$

$\underline{W}_{2}=\frac{1}{\tau_{2}}$ is spontaneous emissions

$W_{p}=\frac{I_{p}}{h v_{p}}\left(\sigma_{p E r}+\sigma_{p Y b}\right)=\mathrm{W}_{\mathrm{pEr}}+\mathrm{W}_{\mathrm{pYb}}=\frac{I_{p}}{h v_{p}} \sigma_{p}$ is the

rate coefficient pumped

$W_{a}=\frac{I_{s}}{h v_{\lambda}} \sigma_{a E r}$ is the rate coefficient of stimulated

absorption

$W_{e}=\frac{I_{s}}{h v_{\lambda}} \sigma_{e E r}$ is the rate coefficient of stimulated

emission

$I_{p}, I_{S}$, is intensity of pumping and signal radiation

$\sigma_{p E r}, \sigma_{a E r}, \sigma_{e E r}$ are the pumping, absorption and emission effective cross sections for erbium

\section{$h$ is Planck's constant}

In a three-level system, the highest level of E3 together with the base level E1 forms the transition allowing the absorption of exciting radiation. After E3 excitation, the quantum system is ideally switched to E2 immediately. Thus, the E3 level is practically zero, and since it does not directly participate in the amplification of the signal, it is not considered in the model described below.

For $N_{3}=0$ it is possible formulate (8) and (9)

$\frac{d N_{2}}{d t}=W_{p} N_{1}+W_{a} N_{1}-W_{2} N_{2}-W_{e} N_{2}$

$N_{t o t}^{E r+Y b}=N_{1}+N_{2}=N_{t o t}$

Static solution of rate equations

$\mathrm{N}_{2}=\frac{\left(W_{p}+W_{a}\right) N_{t o t}^{e r} \tau_{2}}{1+\tau_{2}\left(W_{p}+W_{a}+W_{e}\right)}$

Equation (11) of optical signal propagation by optical waveguide 
$\frac{d I_{s}(z)}{d z}=I_{s}\left(\sigma_{e}(v) N_{2}-\sigma_{a}(v) N_{1}\right)$

Equation (12) of propagation of pumping radiation:

$\frac{d I_{p}(z)}{d z}=\sigma_{e}(v) N_{1} I_{p}$

In general is : $I(z)=\frac{P(z)}{S}=\frac{P(z) \Gamma}{\pi r^{2}}$

It is advantageous to describe the signal gain in the waveguide by optical power $\mathrm{P}(\mathrm{z})$ rather than intensity:

$P(z)=I(z) \pi r^{2} / \Gamma$

In addition, the resulting relationships for calculating the waveguide gain are given depending on its length and the required pumping power that were derived under certain simplified assumptions.

Rate equations: $\frac{N_{2}}{N_{t}}=\frac{\sum_{\lambda} \frac{P_{\lambda}(z) \alpha_{\lambda}}{h \nu \xi}}{1+\sum_{\lambda} \frac{P_{\lambda}(z)\left(\alpha_{\lambda}+g_{\lambda}\right)}{h \nu \xi}}$

$\alpha_{\lambda}=\sigma_{a, \lambda} \Gamma_{\lambda} N_{t}, g_{\lambda}=\sigma_{e, \lambda} \Gamma_{\lambda} N_{t}$ where $N_{t}$ is average (16)

concentration of doping

where $\alpha_{\lambda}, g_{\lambda}$ are coefficients of attenuation and gain.

$\Gamma_{\lambda}$ is the overlap integral (overlap factor - does not depend on $z$ ) with which the effective average radiation intensity in the doped activator region can be expressed.

The gain of a waveguide $G_{d B}(\lambda)$ in [dB] depending on its length is described by equation (17):

$G_{d B}(\lambda)=10 \log _{10}(e)\left[\left(\alpha_{\lambda}+g_{\lambda}\right) \frac{N_{2}}{N_{t o t}}-\left(\alpha_{\lambda}+\alpha_{\lambda 0}\right)\right] \cdot \mathrm{L}$

The gain $G_{d B}(\lambda)$ of the waveguide expressed by the $\mathrm{Wa}, \mathrm{We}, \mathrm{Wp}$ rate coefficients is given by the equation (18)

$$
\begin{aligned}
& G_{d B}(\lambda)=10 \log _{10}(\mathrm{e})\left[\left(\alpha_{\lambda}+g_{\lambda}\right) \frac{W_{a}+W_{p}}{\frac{1}{\tau_{2}}+W_{p}+W_{a}+W_{e}}-\right. \\
& \left.\left(\alpha_{\lambda}+\alpha_{\lambda 0}\right)\right] \cdot L
\end{aligned}
$$

\subsection{Rate Equations for Three-Level System for $\mathrm{Er}^{3+}$ lons and BAC-Ge}

Figure 11 shows a possible arrangement of a three-level energy system of the $\mathrm{Er}$ and BAC-Ge complex. It is known that the optical properties of bismuth-doped glass strongly depend on the chemical composition of the glass resp. on the modifiers used, which in this case were $\mathrm{Ge}$ and $\mathrm{Si}$. In particular, the absorption band BAC-Ge significantly overlaps with the absorption band $\mathrm{Er}^{3+}$. Thanks to this overlap, it is possible to excite both active centers by pumping at one wavelength of $1460 \mathrm{~nm}$. During this excitation, which falls into the two corresponding absorption bands shown in Figure 11 (right), $\mathrm{Er}^{3+}$ and BAC-Ge simultaneously fill the metastable ${ }^{4} I_{13 / 2}$ level and the E2 level. This is an indisputable advantage compared to a situation where two pumping sources are required.

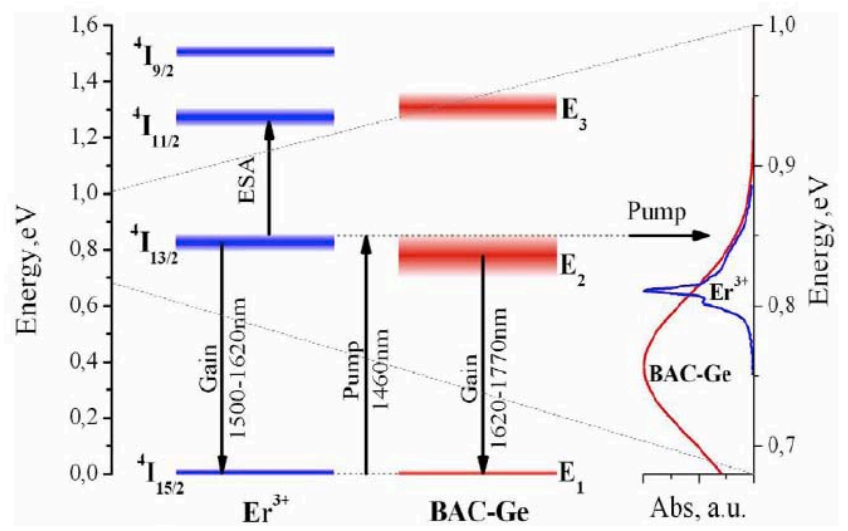

Figure 11: Energetic levels of $\mathrm{Er}^{3+}$ and $\mathrm{BAC}-\mathrm{Ge}$. Absorption bands $\mathrm{Er}^{3+}$ and BAC-Ge (right) [3].

The $\mathrm{Er}^{3+}$ and BAC-Ge complex can roughly be described as a three-level quantum system using rate equations (17) - (19) in the form:

$\frac{d N_{3}}{d t}=-W_{32} N_{3}-W_{31} N_{3}+W_{p} N_{1}$

$\frac{d N_{2}}{d t}=W_{32} N_{3}-W_{2} N_{2}-W_{e E r} N_{2}-W_{e B A C-G e} N_{2}+$

$+W_{a E r} N_{1}+W_{a B A C-G e} N_{1}+W_{p} N_{1}$

$N_{\text {tot }}^{E r+B A C-G e}=N_{1}+N_{2}+N_{3}=N_{t o t}$

where $W_{32}$ is the rate coefficient of non-radiation transition from $E_{3}$ to $E_{2}$,

$W_{31}$ is the rate coefficient of non-radiation transition from $E_{3}$ to $E_{1}$,

$W_{2}=\frac{1}{\tau_{2}}$ is coefficient spontaneous emission,

$W_{p}=\frac{I_{p}}{h v_{p}}\left(\sigma_{p E r}+\sigma_{p B A C-G e}\right)=\mathrm{W}_{\mathrm{pEr}}+\mathrm{W}_{\mathrm{pBAC}-\mathrm{Ge}}=$

$\frac{I_{p}}{h v_{p}} \sigma_{p}$ is the rate pumping coefficient,

$W_{a E r}=\frac{I_{S}}{h v_{\lambda}} \sigma_{a E r}$ is the rate coefficient of stimulated absorption Er,

$W_{a B A C-G e}=\frac{I_{S}}{h v_{\lambda}} \sigma_{a B A C-G e}$ is the rate coefficient of stimulated absorption BAC-Ge,

$W_{e E r}=\frac{I_{S}}{h v_{\lambda}} \sigma_{e B A C-G e}$ is the rate coefficient of stimulated emission Er,

$W_{e B A C-G e}=\frac{I_{s}}{h v_{\lambda}} \sigma_{e B A C-G e}$ is the rate coefficient of stimulated emission BAC-Ge.

To calculate the BAC emission sections, Fuchtbauer-Ladenburg [2] proposed a relationship 
based on the fact that stimulated emission cross section $\sigma(\lambda)$ as a function of wavelength to a first approximation coincides with the spontaneous emission spectrum, which is expressed by the following formula (28),

$\sigma(\lambda)=\frac{\lambda^{2} g(\lambda)}{8 \pi n^{2} \tau}$

where $\lambda$ is wavelength, $g(\lambda)$ is the normalized spontaneous emission shape function, $n$ is the host refractive index, and $\tau$ is the emission lifetime.

By assuming a Gaussian-shaped emission band, the stimulated emission cross section $\sigma$ at the band center can be estimated by the following formula (29)

$\sigma(\lambda)=\frac{\lambda^{2}}{4 \pi n^{2} \tau \Delta v} \sqrt{\ln 2 / \pi}$

\section{Where}

$\lambda$ is the central wavelength of the BAC emission band,

$\Delta v$ is the width of the BAC emission band,

$\tau$ is the lifetime of BAC luminescence,

$n$ is the core refractive index of the waveguide core.

As in the previous case, it is possible express the gain $G_{d B}\left(\lambda_{E r}\right)$ by (30), (31) for for active waveguide $\mathrm{Er}^{3+}$ ions and gain $G_{d B}\left(\lambda_{B A C-G e}\right)$ for BAC-Ge by (32), (33)
$G_{d B}\left(\lambda_{E r}\right)=10 \log _{10}(\mathrm{e})\left[\left(\alpha_{\lambda E r}+g_{\lambda E r}\right) \frac{N_{2}}{N_{t}}-\left(\alpha_{\lambda E r}+\right.\right.$ $\left.\left.\alpha_{\lambda 0 E r}\right)\right] \cdot \mathrm{L}$

The gain $G_{d B}\left(\lambda_{E r}\right)$ of the waveguide expressed by the $W_{a E r}, W_{e E r}, W_{p E r}$ rate coefficients is given by (29), $G_{d B}\left(\lambda_{E r}\right)=10 \log _{10}(\mathrm{e})\left[\left(\alpha_{\lambda E r}+g_{\lambda E r}\right)\right.$.

$\left.\frac{W_{a E r}+W_{p E r}}{\frac{1}{\tau_{2}}+W_{p E r}+W_{a E r}+W_{e E r}}-\left(\alpha_{\lambda E r}+\alpha_{\lambda 0 E r}\right)\right] \cdot L$

and for BAC-Ge by (30)

$G_{d B}\left(\lambda_{B A C-G e}\right)=10 \log _{10}(\mathrm{e})\left[\left(\alpha_{\lambda B A C-G e}+g_{\lambda B A C-G e}\right) \frac{N_{2}}{N_{t}}-\right.$

$\left.\left(\alpha_{\lambda B A C-G e}+\alpha_{\lambda 0 B A C-G e}\right)\right] \cdot \mathrm{L}$

The gain of the waveguide expressed by the $W_{a B A C-G e}, W_{\mathrm{eBAC}-\mathrm{Ge}}, W_{\mathrm{pBAC}-\mathrm{Ge}}$, rate coefficients is expressed by (31)

$G_{d B}\left(\lambda_{B A C}\right)=10 \log _{10}(\mathrm{e})\left[\left(\alpha_{\lambda B A C-g e}+g_{\lambda B A C-G e}\right)\right.$

$\frac{W_{a B A C-G e}+W_{p B A C-G e}}{\frac{1}{\tau_{2}}+W_{p B A C-G e}+W_{a B A C-G e}+W_{e B A C-G e}}-\left(\alpha_{\lambda B A C-G e}+\right.$
$\left.\left.\alpha_{\lambda 0 B A C-G e}\right)\right] \cdot \mathrm{L}$

$L$ is the length of the active waveguide

These relationships apply approximately results, the exact solution requires a numerical solution of the mathematical model (17) - (19). However, the approximate solutions and simplicity of the calculation

Table 2: Emission Parameters of Luminescence Spectra Under Single $830 \mathrm{~nm}, 980 \mathrm{~nm}$ and $1480 \mathrm{~nm}$ Pumping

\begin{tabular}{|c|c|c|c|c|}
\hline Pump & BAC-AI & $\Delta \mathbf{v}$ & $\mathbf{T}$ & $\sigma_{\mathrm{e}-\mathrm{Al}}$ \\
\hline$[\mathrm{nm}]$ & {$\left[\lambda_{c}-n m\right]$} & [nm] & $\mu \mathbf{s}$ & $\mathrm{cm}^{2}$ \\
\hline 830 & 1100 & 193.1 & 460 & $2.81 \times 10^{-2}$ \\
\hline 980 & 1125 & 202.5 & 844 & $1.70 \times 10^{-2}$ \\
\hline 1480 & $\ldots$ & $\ldots$ & $\ldots$ & $\ldots$ \\
\hline Pump & BAC-P & $\Delta \mathbf{v}$ & $\mathbf{T}$ & $\sigma_{\text {e-P }}$ \\
\hline [nm] & {$[\lambda-\mathrm{nm}]$} & {$[\mathrm{nm}]$} & $\mu s$ & $\mathrm{~cm}^{2}$ \\
\hline 830 & 1315 & 131.9 & 564 & $2.3 \times 10^{-21}$ \\
\hline 980 & 1350 & 200.2 & 624 & $3.2 \times 10^{-21}$ \\
\hline 1480 & $\ldots$ & $\ldots$ & $\ldots$ & $\ldots$ \\
\hline Pump & BAC-Si & $\Delta \mathbf{v}$ & $\mathbf{T}$ & $\sigma_{\mathrm{e}-\mathrm{Si}}$ \\
\hline [nm] & {$[\lambda-\mathrm{nm}]$} & [nm] & $\mu s$ & $\mathrm{~cm}^{2}$ \\
\hline 830 & 1425 & 110.7 & 626 & $2.1 \times 10^{-21}$ \\
\hline 980 & $\ldots$ & $\ldots$ & $\ldots$ & $\ldots$ \\
\hline 1480 & $\ldots$ & $\ldots$ & $\ldots$ & $\ldots$ \\
\hline Pump & BAC-Ge & $\Delta \mathbf{v}$ & $\mathbf{T}$ & $\sigma_{e-G e}$ \\
\hline [nm] & {$[\lambda-\mathrm{nm}]$} & [nm] & $\mu s$ & $\mathrm{~cm}^{2}$ \\
\hline 830 & 1430 & 180 & 530 & $3.9 \times 10^{-21}$ \\
\hline 980 & 1560 & 190 & 540 & $4.8 \times 10^{-21}$ \\
\hline 1480 & 1610 & 240 & 490 & $7.6 \times 10^{-21}$ \\
\hline
\end{tabular}


Table 3a: Measured and Calculated Gain of Active Waveguide with Doping Parameters \# 1

\begin{tabular}{|c|c|c|c|c|}
\hline fiber \# & wavelength $\boldsymbol{\lambda}[\mathrm{nm}]$ & $\mathbf{G}_{\text {meas }}[\mathrm{db} / \mathrm{m}]$ & $\mathbf{G}_{\text {calc }}[\mathrm{dB} / \mathrm{m}]$ & Deviation $\mathbf{G}_{\mathrm{d}}[\mathrm{dB}]$ \\
\hline \hline$\# 1$ & 1535 & 0,4 & 0,48 & $-0,08$ \\
\hline$\# 1$ & 1550 & 0,3 & 0,4 & $-0,1$ \\
\hline$\# 1$ & 1600 & 0,2 & 0,25 & $-0,05$ \\
\hline$\# 1$ & 1675 & 0,6 & 0,49 & 0,11 \\
\hline$\# 1$ & 1700 & 0,55 & 0,85 & $-0,3$ \\
\hline
\end{tabular}

Table 3b: Measured and Calculated Gain of Active Waveguide with Doping Parameters \# 2

\begin{tabular}{|c|c|c|c|c|}
\hline fiber \# & wavelength $\boldsymbol{\lambda}[\mathrm{nm}]$ & $\mathbf{G}_{\text {meas }}[\mathrm{db} / \mathrm{m}]$ & $\mathbf{G}_{\text {calc }}[\mathrm{dB} / \mathrm{m}]$ & Deviation $\mathbf{G}_{\mathrm{d}}[\mathrm{dB}]$ \\
\hline \hline$\# 2$ & 1535 & 0,65 & 0,71 & $-0,06$ \\
\hline$\# 2$ & 1550 & 0,51 & 0,64 & $-0,13$ \\
\hline$\# 2$ & 1600 & 0,2 & 0,15 & 0,05 \\
\hline$\# 2$ & 1675 & 0,16 & 0,15 & 0,01 \\
\hline$\# 2$ & 1700 & 0,2 & 0,35 & $-0,15$ \\
\hline
\end{tabular}

Table 3c: Measured and Calculated Gain of Active Waveguide with Doping Parameters \# 3

\begin{tabular}{|c|c|c|c|c|}
\hline fiber \# & wavelength $\boldsymbol{\Lambda}[\mathrm{nm}]$ & $\mathbf{G}_{\text {meas }}[\mathrm{db} / \mathbf{m}]$ & $\mathbf{G}_{\text {calc }}[\mathbf{d B} / \mathbf{m}]$ & Deviation $\mathbf{G}_{\mathrm{d}}[\mathrm{dB}]$ \\
\hline \hline$\# 3$ & 1535 & 4,1 & 3,7 & 0,4 \\
\hline$\# 3$ & 1550 & 2,7 & 2,4 & 0,3 \\
\hline$\# 3$ & 1600 & 0,2 & 0,1 & 0,1 \\
\hline$\# 3$ & 1675 & $\ldots$ & $-0,02$ & $\ldots$ \\
\hline$\# 3$ & 1700 & $\ldots$ & $-0,7$ & $\ldots$ \\
\hline
\end{tabular}

are very useful. The measured and calculated gain of the active waveguide with doping parameters \# 1, \# 2, \# 3 are shown in Tables $\mathbf{3 a}, \mathbf{3 b}, \mathbf{3 c}$.

\section{CONCLUSION}

The article summarizes the existing knowledge about the principles and properties of active waveguides doped with bismuth $(\mathrm{Bi})$ and erbium (Er3 + ). Germaniun was used as a modifier in the case of bismuth, which forms a BAC-Ge complex with bismuth. Furthermore, a simplified mathematical model for calculating the gain of active waveguides was developed. It is known [3] that bismuth with an admixture of germanium forms a BAC-Ge complex, whose luminescence spectral characteristic overlaps with the spectral characteristic of erbium and extends it to the longer wavelength range (NIR). This modified structure makes it possible to build ultra-wideband optical amplifiers with a large bandwidth of up to 250 $\mathrm{nm}$ in the area of the third optical attenuation window of silica waveguides. Since bismuth is a transition metal with external d orbitals, it easily interacts with the environment (with matrix atoms). Unlike rare earth elements, where optically active orbitals are well isolated, there is a much greater influence of the surrounding matrix on broadband radiation than in erbium itself. Thus, it is clear that the adjustment of the concentration of individual dopants and modifiers as well as the material of the matrix itself is of key importance for the resulting spectral characteristics of the active waveguide thus formed. As can be seen in [3], the $\mathrm{Er}^{3+}$ and BAC-Ge activators can be pumped at a single wavelength of $1460 \mathrm{~nm}$. Thus, a broadband amplifier with such a doped active waveguide can be pumped by a single source of pump radiation (pump), which is an indisputable advantage. In addition to using germanium as a modifier, other elements such as silicon, aluminium and phosphorus were tested. However, for telecommunication use in the $C+L+U$ band, the use of germanium as a modifier appears to be most advantageous. The profit values given in the cited publication were verified on our mathematical model. The calculation shows a good agreement between the measured and calculated profit values (Table 3). The differences between the measurements 
and the results from our numerical model were up to $10 \%$. The most balanced spectral characteristic was calculated for the dopant concentrations $\operatorname{Er}(15 \mathrm{ppm})$ and $\mathrm{Bi}$ (140 ppm), where an average gain of $0.4 \mathrm{~dB} / \mathrm{m}$ was achieved. If the length of the active waveguide is 50 meters, then the total gain of the amplifier will be up to $20 \mathrm{~dB}$.

\section{ACKNOWLEDGEMENT}

Our research is supported by the Student Grant Competition of the Czech Technical University in Prague under grant number SGS16/162/OHK3/2T/13.

\section{REFERENCES}

[1] Yan B, Luo Y, Zareanborji A, Xiao G, Peng G-D, Wen J. Performance comparison of bismuth/erbium co-doped optical fiber by $830 \mathrm{~nm}$ and $980 \mathrm{~nm}$ pumping. Journal of Optics 2016; 18: 1-9.

https://doi.org/10.1088/2040-8978/18/10/105705
[2] Zhao Q, Luo Y, Wang W, Canning J, Peng G-D. Enhanced broadband near-IR Luminescence and gain spectra of bismuth/erbium co-doped fiber by 830 and $980 \mathrm{~nm}$ dual pumping. AIP Advances 2017; 7: 045012. https://doi.org/10.1063/1.4981903

[3] Firstov SV, Riumkin KE, Khegai AM, Alyshev SV, Mekumov MA, Khopin VF, Afanasiev FV, Dianov EM. Widebannd bismuth and erbium codoped optical fieber amplifier for $\mathrm{C}+\mathrm{L}$ $+U$ telecommunication band. Laser Phys Lett 2017; 14: 110001.

https://doi.org/10.1088/1612-202X/aa8adf

[4] Murata K, Fujimoto Y, Kanabe T, Fujita H, Nakatsuka. Bi-doped $\mathrm{SiO} 2$ as a new laser material for an intense laser. Fusion Eng Des 1999; 44: 437-9. https://doi.org/10.1016/S0920-3796(98)00334-2

[5] Smejcky J, Jerabek V, Nekvindova P. Gain Determination of Optical Active Doped Planar Waveguides, Conference: Conference on Photonics, Devices, and Systems VII, Prague, AUG 28-30, 2017, Proceedings of SPIE, Volume: 10603, Article 106030P.

https://doi.org/10.1117/12.2294030

[6] Smejcky J, Jerabek V. Nekvindova, Wideband Bismuth Erbium Doped Optical Active Planar Waveguides, Proceedings OK 2018 Prague.

[7] Michel JF. Digonnet, Rare-Earth-Doped Fiber Lasers and Amplifiers. Second Edition, Revised and Expanded.

\section{DOI: https://doi.org/10.31875/2410-4701.2020.07.08}

\section{(C) 2020 Odhiambo et al.; Zeal Press}

This is an open access article licensed under the terms of the Creative Commons Attribution Non-Commercial License (http://creativecommons.org/licenses/by-nc/3.0/) which permits unrestricted, non-commercial use, distribution and reproduction in any medium, provided the work is properly cited. 